\title{
DWDM Networking Method of the Combination Application of FRA and EDFA
}

\author{
Lei Hu \\ Xi’an Fanyi University, Xi’an, China \\ Shuhua_y@126.com
}

Keywords: EDFA, fiber Raman amplifier, WDM, distributed Raman amplifier

\begin{abstract}
Nowadays, the broadband demand increases dramatically, therefore, the optical fiber communication core network have to develop for larger capacity and longer distance. In view of the construction of DWDM(Dense Wavelength Division Multiplexing) transmission system, the combination of FRA(Fiber Raman Amplifier) and EDFA(Erbium Doped Fiber Amplifier) is proposed for the networking of DWDM transmission system, so as to solve the problems of the large capacity and long distance of optical fiber communication core network. By comparing and analyzing the transmission performance between EDFA and FRA, the DSF(Dispersion Shifted Fiber) is adopted, and the DWDM system structure is composed of DRA(Distributed Fiber Raman Amplifier) and EDFA. The application shows that the Raman gain and EDFA gain are flat, and all channel error rates reach $10^{-9}$.
\end{abstract}

\section{Introduction}

With the development of high speed data communication, high quality video communication and multimedia services, the communication capacity of long distance optical transmission system has multiplied, resulting in a large number of commercial applications of WDM(Wavelength Division Multiplexing) technology. The construction of WDM can not be separated from EDFA, and it is also the application of EDFA that enables the fiber communication rate to reach $\mathrm{Tb} / \mathrm{s}$ from the original $10 \mathrm{~Gb} / \mathrm{s}$. If the number of multiplexed wavelengths continues to increase, then EDFA is powerless. Considering the new requirements of optical fiber amplifier, the full band amplification characteristic of FRA can provide the transmission performance guarantee for the networking of DWDM optical fiber communication system ${ }^{[1,2]}$.

\section{Comparison of Performance between EDFA and FRA}

\subsection{EDFA Performance Analysis}

\subsubsection{Saturated gain performance}

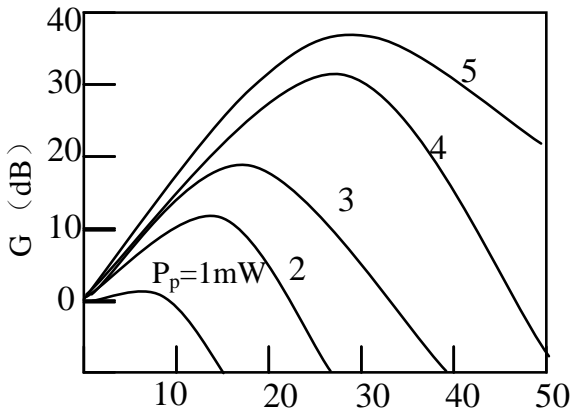

Figure 1. The length of the amplifier (m)

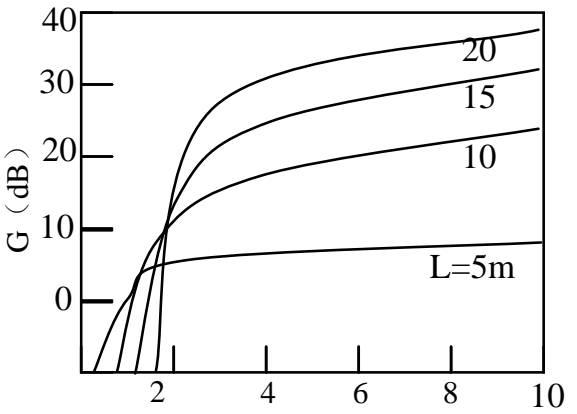

Figure 2. Pump power (mW)

Fig. 1 is the relationship curve between EDFA small-signal gain(G) and the pump power $P_{P}$ and 
the length $l$ of EDFA. The gain coefficient has an optimal value with the length $l$ of the amplifier.

As shown in Fig.2, when the length of the fiber is fixed, it is not that the greater the pump power, the greater the gain coefficient, it has a saturation value.

\subsubsection{Noise characteristic}

The definition of the noise factor $F_{n}$ is $F_{n}=\frac{(S N R)_{\text {in }}}{(S N R)_{\text {out }}}$. The noise factor is used to describe the deterioration of the amplifier to the SNR(signal-to-noise ratio). The smaller the noise factor, the higher the output SNR.

The noise factor of EDFA is related to the population inversion difference value $\Delta \mathrm{N}$. The better the pump, the bigger the $\Delta \mathrm{N}$ and the smaller the noise. The three-level system under the pump is the limit noise index of EDFA.

\subsection{FRA Performance Analysis}

\subsubsection{Saturated gain performance}

Through studying the fiber SRS(Stimulated Raman Scattering), we can find that the silica optical fiber has a wide Raman gain spectrum (up to 40THz). Assuming that the pump light with a frequency of $\omega_{\mathrm{P}}$ and the signal light with a frequency of $\omega_{\mathrm{S}}$ are coupled to the optical fiber through the wavelength selection, when these two beams of light are transmitted together in the optical fiber, the energy of the pump light is transferred to the signal light by the SRS effect, so that the light signal is amplified. The pump light and the signal light can be input at both ends of the fiber, and the weak signal can also be amplified in the reverse transmission process. The optical amplifier based on the SRS mechanism is called FRA.

For FRA, when the signal power increases and the consumption produced by the pump power transferred to the signal can not be ignored, the pump power will decay continuously during transmission, the amplification speed of signal light will also be limited, and the saturation phenomenon will appear during the amplification process. Assuming that the attenuation coefficient $\alpha_{S}=\alpha_{P}$, the approximate formula of the saturation gain is:

In the formula, $r_{0}=\frac{\omega_{P} P_{S}(0)}{\omega_{S} P_{P}(0)}, G_{\mathrm{A}}$ is the small signal gain coefficient. With the increase of $\mathrm{r}_{0}$ (that is the $P_{S}(0)$ ), the gain will be saturated.When $G_{A} r_{0} \approx 1$, the gain is reduced by half. At this time the signal power is close to the input pump power, and the input pump power can represent the saturated output power of the FRA.

\subsubsection{Noise characteristic}

FRA is usually divided into discrete FRA and distributed FRA (DRA). DRA is the process of obtaining distributed gain, so its equivalent noise ratio is smaller than the separate amplifier, and the concentrated noise index can be less than $3 \mathrm{~dB}$, or even negative, which effectively increases the transmission distance of the WDM system. Therefore, the DRA auxiliary transmission improves the performance of WDM system. For the $1530 \mathrm{~nm}$ channel, the signal-to-noise ratio of the system is increased by $4.5 \sim 6.5 \mathrm{~dB}$, and the equivalent noise index $\mathrm{F}_{\mathrm{n}}$ is reduced to $5.9 \sim 8.9 \mathrm{~dB}$.

\subsection{Performance Comparison}

EDFA advantages: Its working wavelength is consistent with the minimum loss window of the fiber. The coupling efficiency is high, and it is easy to couple with fiber. The loss can be reduced to $0.1 \mathrm{~dB}$, and the fusion and reflection loss is very small. It is not easy to self excitation. It has high gain and high output power, that is, the output power can reach $14 \mathrm{dBm}$ in one-way pump, and can reach $17-20 \mathrm{dBm}$ in two-way pump, and the noise factor can be as low as 3-4dB.

FRA advantages: The gain wavelength is determined by the wavelength of the pump light. In fact, it can not only work in C band, but also work in S band (1350-1450nm) and L band (1564-1620nm) to 
meet the requirement of full wave window. The gain medium is the transmission fiber itself, such as DRA, which can make the distributed amplification along the transmission fiber, and the signal power of all parts in the optical fiber is relatively small, which can effectively reduce the influence of all kinds of optical fiber nonlinear effects. The noise index is low. The combined use of DRA and EDFA can significantly increase the total gain of the long distance optical communication system and improve the system $\mathrm{Q}$ value, and the transmission distance of the system is the largest. The gain spectrum is wide, and the effective gain of the $40 \mathrm{~nm}$ range can be realized by single wavelength pump on $\mathrm{DSF}^{[3-6]}$.

\section{The Application of EDFA and FRA in the DWDM System}

\subsection{The Application of EDFA in DWDM System}

EDFA is used to extend the relay distance. Using it in the DWDM system (C and L bands) can achieve the super large capacity. EDFA achieves the magnification of 16, 32, 64, or more wavelength systems in the $\mathrm{C}$ band of the DWDM system. Figure 3 is the application of EDFA in the DWDM system. The EDFA is connected to the output end of the light transmitter to increase the output power and increase the fiber power. EDFA is used in the receiver preamplifier to effectively improve the receiver sensitivity.

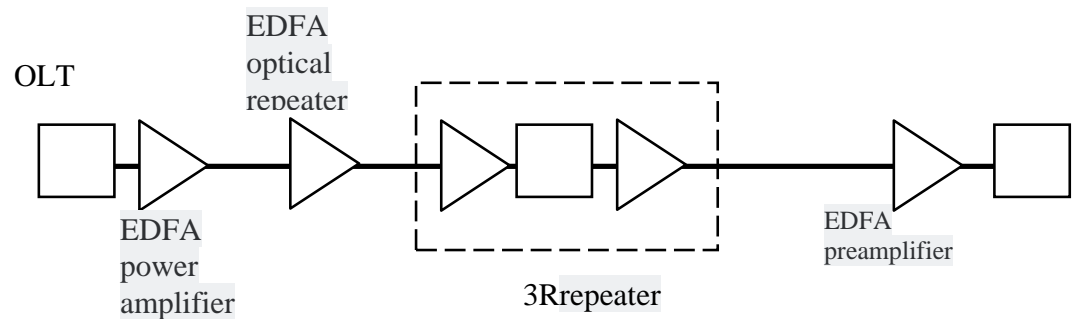

Figure3 The structure of EDFA in DWDM

\subsection{The Application of FRA in DWDM System}

\subsubsection{The application of discrete FRA}

The optical fiber gain medium of discrete FRA is relatively short, and the pump power is from a few watts to dozens of watts. It can generate high gain above 40dB and enlarge the band that EDFA can't enlarge. The configuration: DCF: ordinary fiber is 1: 7, and the pump power is $500 \mathrm{~mW}$.

\subsubsection{The application of distributed FRA}

The transmission performance of the DWDM system is influenced by the nonlinear effects of optical fiber. DRA uses transmission optical fiber as the optical amplification medium, which can reduce the input power of optical fiber, and reduce the nonlinear effects such as FWM(Four Wave Mixing) and XPM(Cross Phase Modulation) as well, so as to avoid the FWM effect and expand the available window of optical fiber.

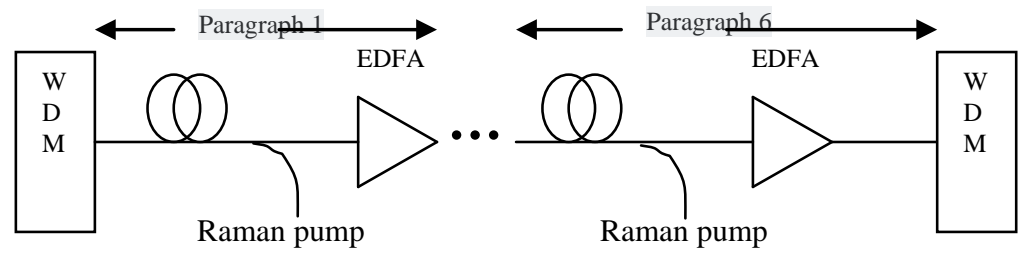

Figure4The structure of DRA+EDFA

The transmission structure using DRA is shown in Fig.4. At each relay section of the WDM system, the reverse Raman pump is injected into the EDFA input end. The signal will implement the distributed FRA along the optical fiber. 


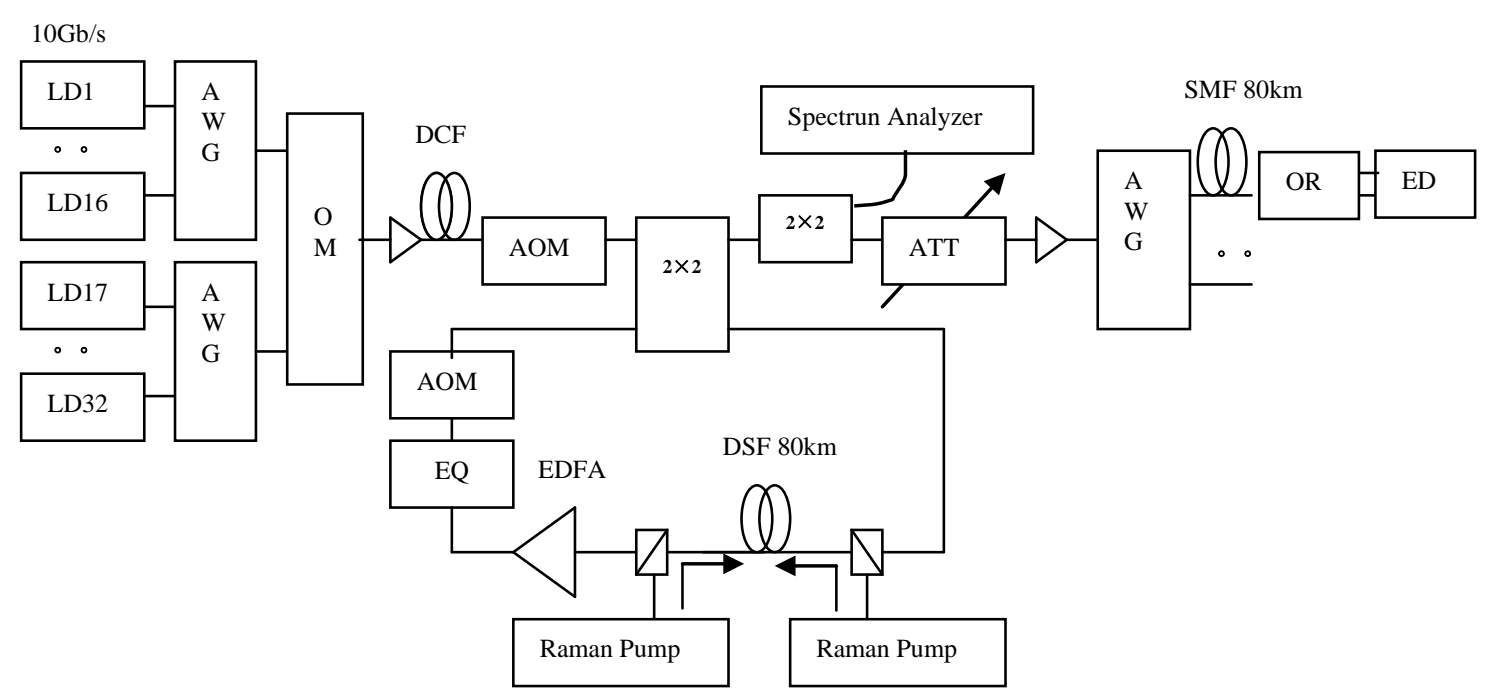

Figure 5. DRA in the DWDM structure

The DRA in the DSF DWDM system is shown in Fig.5. The application distance is $80 \times 8$ kilometers, the transmission capacity is $32 \times 10 \mathrm{~Gb} / \mathrm{s}$ and the channel interval is $50 \mathrm{GHz}$. The wavelength range is 1545.3-1557.8nm, the array waveguide grating (AWG) is used to collect bit stream and the DCF is used to correct the bit flow waveform.

Fig.6 is the gain curve, and the Raman gain is carried out in a two-way pump. The backward pump is higher than forward pump to suppress the nonlinear effect of optical fiber. The forward pump is used to improve the flatness of Raman gain, and the gain of EDFA can suppress the self excited radiation noise. All measured channel error rates reach $10^{-9}$.

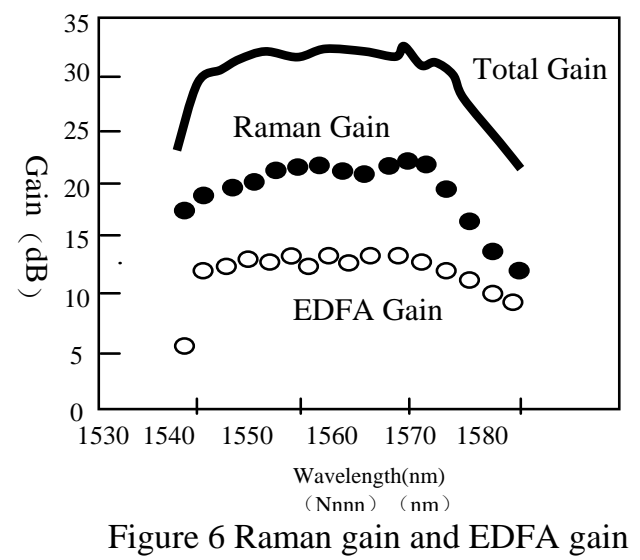

\section{Conclusions}

EDFA has successfully promoted the development of WDM system. EDFA amplifies multiple optical signals for a fiber, and achieves excellent coupling with optical fiber, and has obvious advantages of high gain and low noise. FRA has the advantages of low noise, full band amplification and online amplification by using transmission optical fiber, and the combination of it with EDFA promotes the application of WDM system, especially the DRA technology, which has become the key technology of next generation WDM system. DRA technology can upgrade the existing system to $40 \mathrm{~Gb} / \mathrm{s}$ by improving the spectrum utilization of the WDM system. Through encrypting channel intervals, the system improves the multiplexing degree of transmission, so that the channel interval is upgraded from $100 \mathrm{GHz}$ to $50 \mathrm{GHz}$ without any additional cost. 


\section{Acknowledgement}

In this paper, the research was sponsored by the science research projects of education deparment in Shaanxi provincial government (Project No.16JK2078).

\section{References}

[1] GONG Jia-min, LIU Jian-hua, GUO Cui, et al. Research on Multi-channel Rama Wavelength Conversion Based on Tellurite Fiber [J]. Optical Communication Technology, 2017, 41 (4) :31-34.

[2] ZHOU Zhao, NIE Hai-tao, WANG Yao-jun. Long Reach DWDM-PON with 12.5 GHz Channel Spacing Based on Comb Source Seeding [J]. Optoelectronics Letters, 2016(4):304-307.

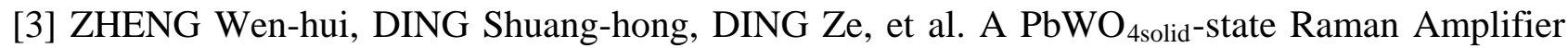
Excited by 1064nm Nan Osecond Pulses[J], Chinese Journal of Lasers[J]. 2014,41:0502011.

[4] ZHANG Meng, LI Hong-kui. Research of The All-solid-state Raman Laser[J]. Journal of Shenyang Ligong University, 2014, 33(2):43-45.

[5] XU Jun-hua,, ZHAO Yun, LENG Bin, et al. All-optical Wavelength Conversion Based on SRS in Optical Fiber [J]. Journal of Applied Optics, 2013, 34 (5):882-888.

[6] PENG Ji-ying, ZHENG Yi. Compact Q-switched and Mode-locked Nd:GdVO 4 self-Raman Laser with Cr:YAG [J]. Journal of Optoelectronics· Laser, 2013, (3):415-418. 